\title{
Quantitative Concepts in Drug Formulation and Absorption and their Relevance for Drug Delivery
}

\author{
Georgios Imanidis*, Marc Sutter, Susanne Reitbauer, Susanne B. Kapitza, Peter van Hoogevest, \\ Daniel Hummel, Beat Müller, and Peter Lütolf
}

\begin{abstract}
A series of theoretical models is presented to describe the processes of drug release and absorption following non-invasive administration by the intestinal and the (trans)dermal route. These models are used to analyze experimental data and provide the means to test hypotheses about the involved mechanisms and influence factors. They thus strengthen the basic understanding of drug release and absorption and help establish quantitative relationships that are relevant for the prediction and control of drug delivery.
\end{abstract}

Keywords: Absorption · Drug delivery $\cdot$ Formulation $\cdot$ Intestinal $\cdot$ Modeling $\cdot($ Trans)dermal

\section{Introduction}

In non-invasive drug administration, appearance of the pharmacologically active substance in the systemic circulation requires that the active substance is released from the delivery system, which may be a dosage form or a device, and is absorbed by the epithelial membrane at the site of administration. For a given drug, the outcome of this two-step process depends on technological formulation factors defined by the delivery system, biological factors arising from the epithelium and, importantly, their mutual interaction. Despite recent advances, the scientific understanding of this process still largely relies on empirical knowledge. The research outlined in this article concentrates on the phenomena that take

\footnotetext{
${ }^{*}$ Correspondence: Prof. Dr. G. Imanidis Institute of Pharmaceutical Technology Department of Pharmaceutical Sciences Pharmacenter, University of Basel

Klingelbergstrasse 50

$\mathrm{CH}-4056$ Basel

Tel.: +41612671513

Fax: +41612671516

E-Mail: georgios.imanidis@unibas.ch
}

place at the interface between the delivery system and the absorptive epithelium, the goal being to elucidate the mechanisms that determine the delivery of the drug into the circulation.

The approach taken in this research utilizes biophysical and mathematical modeling to describe drug release and absorption kinetics. Individual processes are investigated using in vitro experimental models. A key element of the research is the analysis of the experimental data using the theoretically derived models for the purpose of quantitatively assessing the effect of the determining physicochemical and biological factors, and testing the validity of the hypotheses underlying the models. From this, a basic mechanistic understanding of the involved processes may be established The macroscopic modeling is combined with spectroscopic techniques in order to gain an insight into the molecular mechanisms that are responsible for the relevant phenomena.

The ultimate goal of this work is, using a unifying quantitative approach, to develop general relationships for the prediction and consequently control of drug delivery by different routes of non-invasive drug administration taking into account the interrelationship between drug formulation, release and absorption.

Research in the following three areas is presented here: (i) membrane transport in intestinal drug absorption, (ii) topical drug delivery and bioavailability assessment, (iii) transdermal iontophoresis and timecontrolled systemic drug delivery.

\section{Membrane Transport in Intestinal Drug Absorption}

The permeation of drugs through the plasma membrane of epithelial cells is controlled by passive diffusion and carrier-mediated transport processes, both of which may potentially be influenced by the formulation of the delivery system. In addition, drug formulation which entails embedding the drug in a vehicle regulates its release to the epithelial membrane. The objectives of the work were first, to identify the relevant properties of the phospholipid domain of the membrane that influence passive permeability and examine the effect of the formulation on them; second, to delineate the contribution to drug absorption of passive diffusion and carrier-mediated transport taking place in parallel; and last, to determine the effect of the formulation on both diffusion driven and transporter-mediated permeation. The basic understanding gained may then be exploited for the purpose of developing strategies for controlling absorption and delivery.

The average free surface area and the rate of lipid thermal motion in phospholipid bilayers reflecting order and dynamic properties of the membrane, respectively, were identified as relevant properties governing solute permeation. The permeability coefficient $\left(P_{\mathrm{c}}\right)$ of a model solute corrected for partitioning, depended on both the order parameter $(S)$, and the rotational diffusion coefficient $\left(D_{\perp}\right)$ of fluorescent probes inserted into the bilayer membrane according to a theoretical relationship (Eqn. (1)) illustrated in Fig. 1 [1]. 


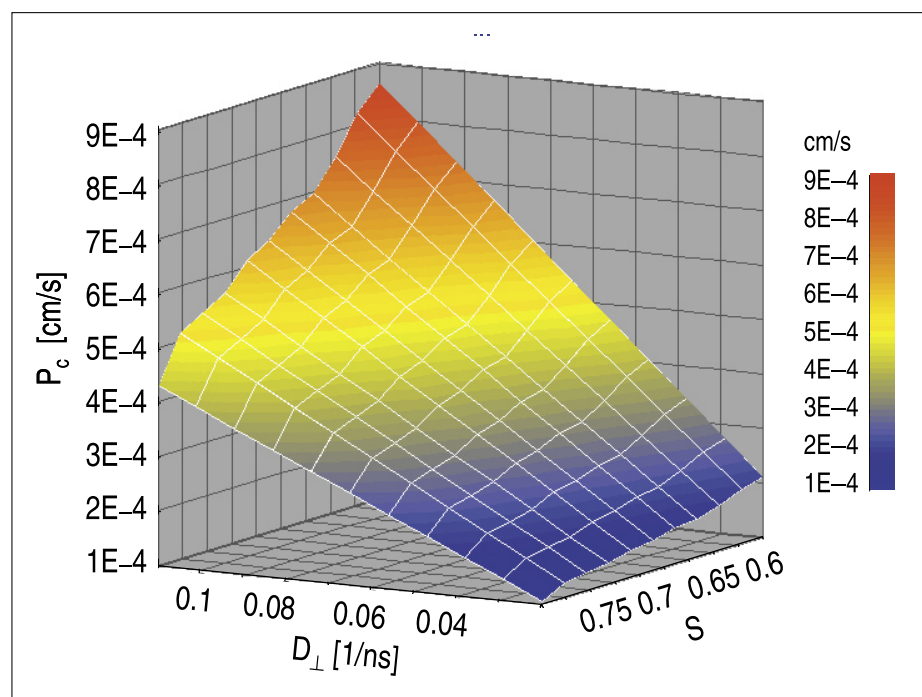

Fig. 1. Permeability coefficient of mannitol used as model solute (corrected for partitioning using an activation energy of $40 \mathrm{~kJ} / \mathrm{mol}$ ) measured in phospholipid vesicles as a function of the order parameter and the rotational diffusion coefficient obtained with TMA-DPH

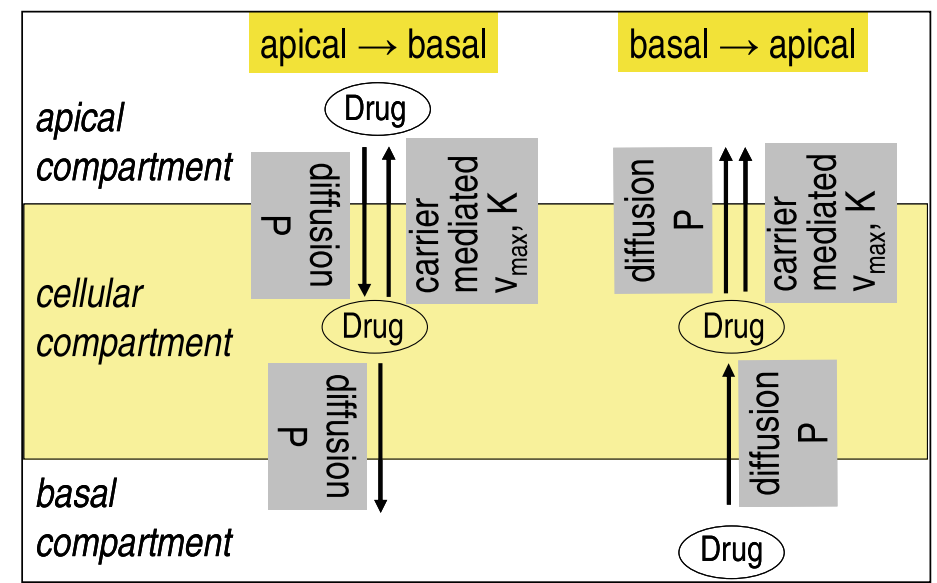

Fig. 2. Transport processes considered for modeling drug permeation in the Caco-2 monolayer and relevant parameters

$$
P_{c}=a D_{\perp} \exp \left(-\frac{b}{3 /(1+2 S)-1}\right)
$$

where,

$$
a=\frac{\alpha \lambda^{2} K_{0}^{\prime}}{2 h}, \quad b=\frac{(\gamma+1) A^{*}}{A_{0}},
$$

$\lambda$ is the length of a diffusive step, $K_{0}^{\prime}$ is the temperature corrected partition coefficient of a point solute, $h$ is the bilayer membrane thickness, $A^{*}$ is the cross-sectional area of the solute, $A_{0}$ is area of a phospholipid molecule, $\gamma$ is a coefficient accounting for the overlap of free surface area, and $\alpha$ is a proportionality constant.

The parameters $S$ and $D_{\perp}$ were determined by time-resolved fluorescence anisotropy measurements with the fluorescent probes DPH and TMA-DPH, and were used to express lipid order and thermal motion, respectively. The latter evoked the redistribution rate of free surface area of the bilayer membrane. Eqn. (1) adequately explained the experimental permeability data [1].

The effect of liposomes on the phospholipid domain of the plasma membrane of adherent epithelial Caco- 2 cell monolayers was assessed by steady-state fluorescence anisotropy and fluorescence life-time measurements. Localization of the fluorescent probes in the cells was ascertained by resonance energy transfer-induced fluorescence quenching. Liposomal formulations can be used as drug vehicles for the peroral delivery of poorly water soluble drugs, improving the dissolution of the drugs in intestinal fluids. Liposomes made of soybean phosphatidyl choline in non-cell toxic concentrations elicited, in a concentration dependent manner, a statistically significant decrease of fluorescence anisotropy and fluorescence life-time of probes incorporated in the plas- ma membrane of Caco- 2 cells. This indicated that this formulation interacted with the epithelium causing a decrease of the order and/or an increase of the mobility in the phospholipid domain of the plasma membrane. This effect was most pronounced in the highly lipophilic core of the plasma membrane where an anisotropy decrease of $30 \%$ was reached [2]. Thus, an increase of the solute permeability of the membrane by passive diffusion may be expected.

The permeation kinetics of drugs that were formulated using liposomes and were subject to carrier-mediated apical efflux in Caco- 2 cell monolayers were analyzed using a theoretical model. This model took into account simultaneous movement of drug between the apical, the basal and the cellular compartment by passive diffusion and saturable carrier-mediated transport (Fig. 2). The change of drug concentration with time in each compartment for apicalto-basal and basal-to-apical direction of permeation was described by the system of Eqns (2) to (7) [3].

$$
\frac{d C_{A a b}}{d t}=-P\left(C_{A a b}-C_{C a b}\right) \frac{S_{m}}{V_{A}}+\frac{V_{\max } m_{C a b}}{K+m_{C a b}} \frac{1}{V_{A}}
$$

$$
\frac{d C_{B a b}}{d t}=P\left(C_{C a b}-C_{B a b}\right) \frac{S_{m}}{V_{B}}
$$

$$
\begin{aligned}
\frac{d m_{C a b}}{d t}= & P\left(C_{A a b}-C_{C a b}\right) S_{m}- \\
& \frac{v_{\max } m_{C a b}}{K+m_{C a b}}-P\left(C_{C a b}-C_{B a b}\right) S_{m}
\end{aligned}
$$

$$
\frac{d C_{A b a}}{d t}=P\left(C_{C b a}-C_{A b a}\right) \frac{S_{m}}{V_{A}}+\frac{v_{\max } m_{C b a}}{K+m_{C b a}} \frac{1}{V_{A}}
$$

$$
\frac{d C_{B b a}}{d t}=-P\left(C_{B b a}-C_{C b a}\right) \frac{S_{m}}{V_{B}}
$$

$$
\begin{aligned}
\frac{d m_{C b a}}{d t}= & P\left(C_{B b a}-C_{C b a}\right) S_{m}- \\
& \frac{v_{\max } m_{C b a}}{K+m_{C b a}}-P\left(C_{C b a}-C_{A b a}\right) S_{m}
\end{aligned}
$$

where $C$ is the concentration, $m$ is the mass, the indices $A, B$, and $C$ (upper case) denote the apical, basal and cellular compartments, respectively, the indices $a b$ and $b a$ (lower case) denote permeation in the apical-tobasal and the basal-to-apical direction, respectively, $S_{\mathrm{m}}$ is surface area of the monolayer, $V$ is volume of compartment, $P$ is the passive permeability coefficient, $v_{\max }$ is the maximum transport rate of carrier-mediated efflux, and $K$ is the mass in the cellular compartment for which one half of $v_{\max }$ is attained, also referred to as the affinity or dissociation constant.

The system of differential equations was fitted to concentration data of transport experiments in the Caco-2 system, and optimal values of the parameters $P, v_{\max }$ and $K$ were deduced using a least-squares procedure and a numerical differentiation algorithm. The passive permeability coefficient of two drugs, Indinavir and Saquinavir, applied with no liposomes was $5.19 \times 10^{-5} \mathrm{~cm} / \mathrm{s}$ and $8.97 \times 10^{-5} \mathrm{~cm} / \mathrm{s}$, respectively, in accordance with their lipophilicity difference and their apparent affinity parameter for efflux carrier(s) $K$ was $0.022 \mu \mathrm{g}$ and $0.0034 \mu \mathrm{g}$, respectively. The efflux inhibitor Vinblastine reduced the apparent efflux carrier affinity $(K=0.489$ 
$\mu \mathrm{g})$ but did not affect the passive permeability coefficient. The liposomal formulations elicited a saturation of apical efflux which assumed a $v_{\max }$ value of $3.72 \times 10^{-3}$ $\mu \mathrm{g} / \mathrm{cm}^{2} / \mathrm{s}$. This was accompanied by an elevated drug amount in the cellular compartment and a passive drug flux equaling or exceeding $v_{\max }$. Also, the liposomal formulation increased the passive flux, i.e. the passive delivery rate, of one of the drugs (Saquinavir) roughly six-fold by a mechanism probably mostly related to the release of drug from the vehicle to the epithelial membrane [3]. This example demonstrates the possibility of using this method for quantitatively delineating diffusional and carrier-mediated transport mechanisms in order to assess the effect of the liposomal formulations on each of them and hence on epithelial permeation.

\section{Topical Drug Delivery and Bioavailability Assessment}

Topical formulations typically consist of several components comprising two or more phases. The quantitative composition and the phases of these formulations change during the time of application as a result of environmental influences. Composition, phase structure and their time-dependent alteration affect drug release and epidermis permeation as well as the interaction of the formulation with the stratum corneum. Furthermore, the availability of the drug at the site of pharmacologic action for dermal therapy depends on the delivery rate of the drug across the skin. The objectives of this work were first, to determine the mode of influence of colloidal and coarse dispersed phases on drug release and identify the pertinent drug concentration of the formulation that drives delivery; sec- ond, to establish the possibility of predicting the effect of time-dependent alteration of the formulation on delivery; and last, to define the relevant drug concentration in cutaneous tissue for pharmacologic activity and hence bioavailability of a dermal delivery system and finally introduce a methodology for determining drug concentration at the target site in the skin and establishing concentration-effect relationships.

The free concentration of drug in the continuous phase of the formulation was found to govern release and skin permeation kinetics. This concentration is given by Eqn. (8) [4].

$$
C_{D}^{\prime \prime}=\frac{C_{D}^{\text {tot }}}{\phi^{\prime}\left(K_{I / I}-1\right)+1}
$$

where $\phi^{\mathrm{I}}$ is the volume fraction of the dispersed phase, $K_{\mathrm{I} / \mathrm{II}}$ is the drug partition coefficient between the dispersed and the continuous phase, and $C_{\mathrm{D}}{ }^{\text {tot }}$ is the total drug concentration in the formulation.

The effect that the dispersed phase(s) of a formulation exerts on the delivery rate could be fully expressed by means of $\phi^{\mathrm{I}}$ and $K_{\mathrm{I} / \mathrm{II}}$. The dependence of the apparent permeability coefficient encompassing drug release and skin permeation on $C_{\mathrm{D}}^{\mathrm{II}}$ is illustrated by Fig. 3 .

The alteration of the formulation as a function of application time arising from the evaporation of volatile components was expressed in terms of the change inflicted upon $C_{\mathrm{D}}$ II , which depended on the volume fraction $\phi^{\mathrm{I}}$ and the partition coefficient $K_{\mathrm{I} / \mathrm{II}}$ according to Eqn. (9) (infinite dose).

$$
\frac{C_{D}^{\prime \prime}(t)}{C_{D}^{\|}(0)}=\frac{\left(K_{I I I}-1\right)+1 / \phi^{\prime}(0)}{\left(K_{I / I}-1\right)+1 / \phi^{\prime}(t)}
$$

Changes in the delivery rate as a result of alteration of the formulation were consistently explained based on $C_{\mathrm{D}}{ }_{\mathrm{D}} \mathrm{II}(\mathrm{t})$ further supporting the validity of this approach for evaluating dermal and transdermal delivery [4].

The epidermal/dermal junction was postulated to be the site of pharmacologic action of many drugs in the skin, since it is adjacent to the metabolically active basal cell layer and to the vascularized dermal papillae with their population of cells involved in the immune response. Drug concentration at this target site can be estimated from the concentration profile in different layers of cutaneous tissue, illustrated in Fig. 4. Aqueous concentration (termed $C^{*}$ ) of unbound drug at this site achieved by dermal application was estimated using Eqn. (10), which was derived based on the model depicted on Fig. 4 [5][6]. Eqn. (10) employs in vitro-measured permeability coefficients of the control membrane, $P_{\text {contr }}$, and of the dermis, $P_{\text {der/20 }}$, the latter being adjusted to the in vivo effective thickness for diffusion, and the pertinent drug concentration $\left(C_{\mathrm{D}}{ }_{\mathrm{II}}\right)$ in the formulation.

$$
C^{*}=\frac{P_{\text {contr }} \cdot C_{D}^{\prime \prime}}{P_{\text {der } / 20}}
$$

Calculated $C^{*}$ concentrations of nicotinic acid derivatives applied at greatly differing concentrations provided nearly identical correlation patterns with the pharmacologic effect (inflammation, vasodilation) of the drugs in vivo (Fig. 5) [5]. In a separate study, $C^{*}$ was shown to correlate with the antiviral effect of Acyclovir in the treatment of cutaneous viral infections [6]. These studies validate $C^{*}$ as the relevant target concentration for the

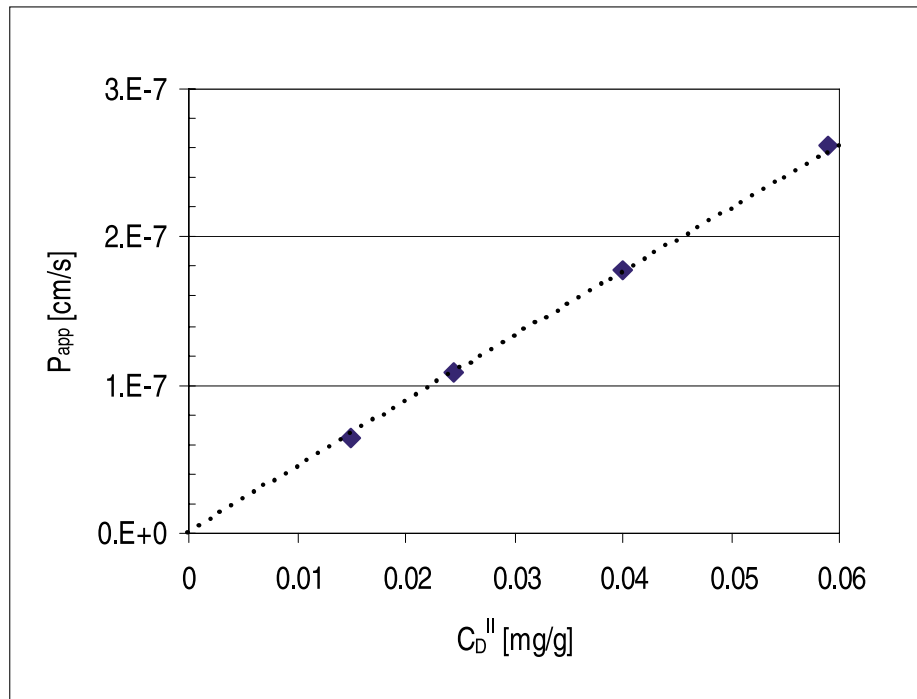

Fig. 3. Apparent permeability coefficient of different dermal formulations of S-Ibuprofen for pig ear skin as a function of the free drug concentration in the continuous phase

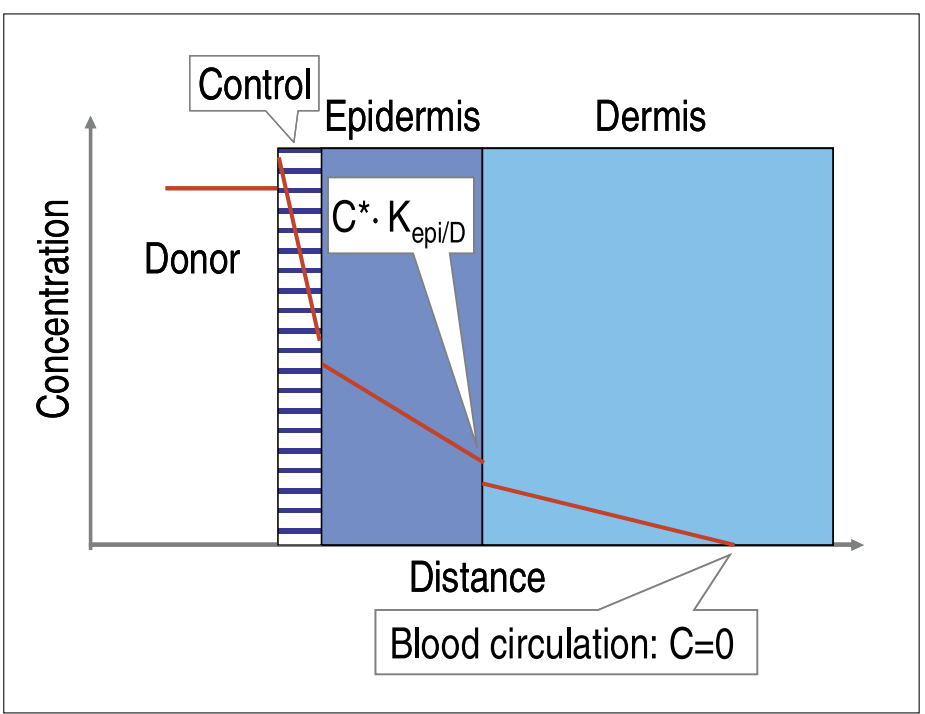

Fig. 4. Drug concentration profile in the skin under steady-state flux conditions for application of a formulation (donor) onto the surface. $K_{\mathrm{epi} / \mathrm{D}}$ is partition coefficient 


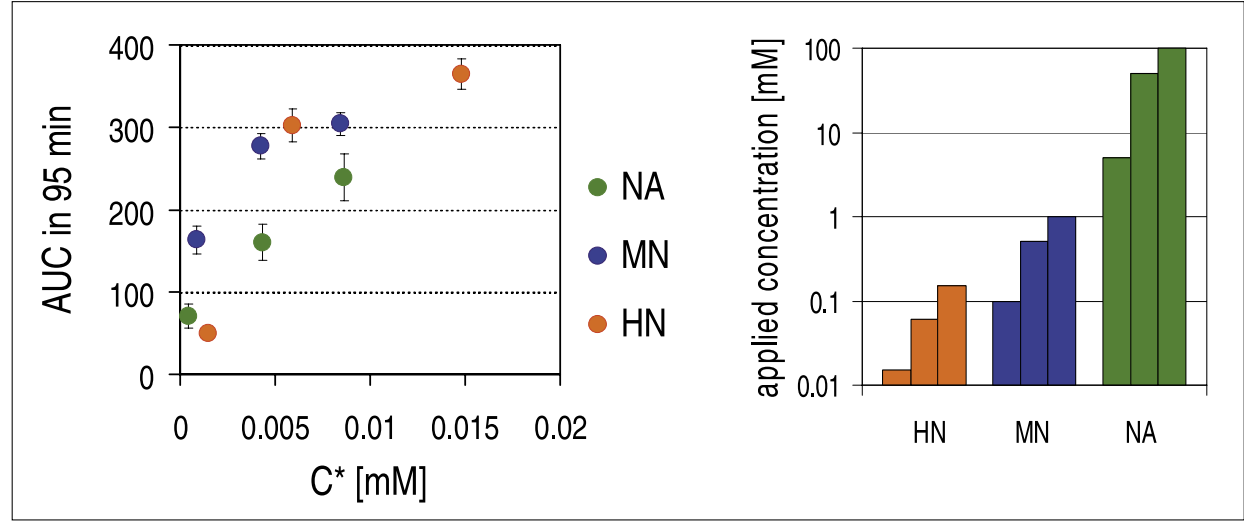

Fig. 5. Vasodilation measured in vivo as cumulative erythema using a chromameter as a function of site of action concentration in the skin (left panel) for nicotinic acid (NA) methyl nicotinate (MN) and hexyl nicotinate $(\mathrm{HN})$. Right panel shows drug concentrations applied as aqueous solution to the skin.

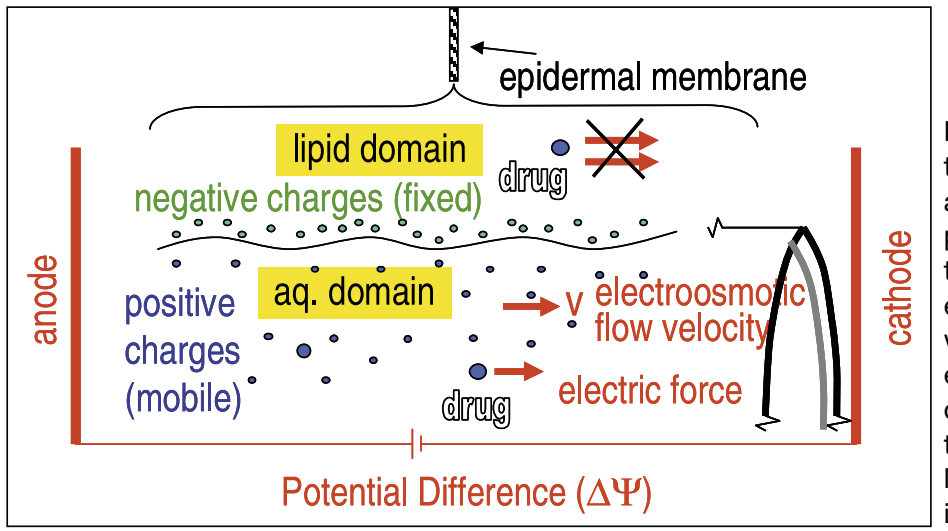

Fig. 6. Electrochemical transport processes of a drug in the aqueous pore domain and the lipid domain of epidermal membrane with application of an electric voltage. The ion distribution comprising the electrical double layer at the domain interface is indicated.

pharmacologic effect and bioavailability in the skin.

\section{Transdermal lontophoresis and Time-controlled Systemic Drug Delivery}

The inherently low rate of delivery of drugs across the skin may be enhanced by the application of an electric voltage to the skin, i.e. by iontophoresis. This voltage may also be used to control the delivery rate in real-time. Time-controlled delivery is required for drugs applied transdermally in order to attain optimized pharmacodynamics. Achieving enhancement and regulation of transdermal delivery by passive diffusion, if possible, would complement iontophoresis while avoiding its side-effects. The objectives of this study were first, to develop a theoretical model for the enhancement factor reached by iontophoresis taking into account the lipophilicity-dependent lipid-to-aqueous pathway permeation, the ionic valence of the drug in the aqueous permeation pathway of the epidermis, the convective transport by electroosmosis, and the tissue retention of the drug and then use this model to analyze experimental data of different permeants in order to quantify the impact of these factors on iontophoretic enhancement and set up a strategy for predict- ing enhancement. Second, was to develop a method based on passive diffusion for regulating delivery and controlling it in an on/off fashion.

An expression for the enhancement factor, $E$, (Eqn. (11)) was derived based on the Nernst-Planck equation for an electrical potential difference $\Delta \Psi$ applied to the epidermal membrane of thickness $h$, with $P_{\mathrm{ld}}$ and $P_{\text {ad }}$ being the passive drug permeability coefficients of the lipid and aqueous permeation pathway of the membrane, respectively, $\in$ and $\in$ ' being the surface area fraction of the aqueous domain under passive and iontophoretic conditions, respectively.

$$
E=\frac{\frac{\epsilon /\left(-\bar{\epsilon}_{a d} B+P e\right)}{1+\left(P_{l d} / P_{a d}\right)^{p a s s i v e}}}{1-\exp \left(-\frac{-\bar{Z}_{a d} B+P e}{1+\left(P_{l d} / P_{a d}\right)^{p a s s i v e}}\right)}
$$

where,

$\bar{z}_{a d}=\alpha_{a d, 1} z_{1}+\alpha_{a d, 2} z_{2}$

$B=\frac{F \Delta \Psi}{R T}$

$P e=\frac{v h}{D_{a d}}$
The model incorporated the effect of the electrical double layer at the lipid/ aqueous domain interface (shown in Fig. 6 for a positively charged drug at physiological $\mathrm{pH}$ ) on electroosmotic flow velocity $(v)$ and on the weighted average net ionic valence $\bar{z}_{a d}$ of the drug in the aqueous pathway estimated using the Poisson-Boltzmann equation. Analysis of the data of a number of low molecular weight compounds (including peptides) using Eqn. (11) provided consistent results on the contribution of the individual factors to the permeation enhancement of the drugs, lending support to the model [7-9].

Under conditions involving solely passive diffusion, it was possible to deliver transdermally in vitro nicotine and nitroglycerine in repeated on/off cycles each lasting between 6 and $12 \mathrm{~h}$ using a proprietary technology developed in this laboratory [10] (owner ChronoTherapeutics Inc., NY, USA). Delivery rate was controlled within the required range for attaining therapeutic effects. With this technology, which can be automated and miniaturized, delivery of a drug at various times of the day and prevention of tolerance development could possibly be achieved. A prototype device is currently under development aiming at commercialization with an industrial partner.

Received: December 29, 2005

[1] M. Sutter, T. Fiechter, G. Imanidis, $J$. Pharm. Sci. 2004, 93, 2090.

[2] S. Reitbauer, $\mathrm{PhD}$ thesis, University of Basel, 2005.

[3] S.B. Kapitza, B.R. Michel, P. van Hoogevest, M.L.S. Leigh, G. Imanidis, Eur. $J$. Pharm. Biopharm. 2005, in press.

[4] D. Hummel, G. Imanidis, in 'The Essential Stratum Corneum', Eds. R. Marks, J-L. Leveque, R. Voegeli, Martin Dunitz, London, 2002, p. 119.

[5] B. Müller, M. Kasper, C. Surber, G. Imanidis, Eur. J. Pharm. Sci. 2003, 20, 181.

[6] G. Imanidis, W-Q. Song, P.H. Lee, M-H. Su, E.R. Kern, W.I. Higuchi, Pharm. Res. 1994, 11, 1035.

[7] C. Kochhar, G. Imanidis, J. Pharm. Sci. 2003, 92, 84.

[8] G. Imanidis, P. Luetolf, J. Pharm. Sci., in press.

[9] M. Altenbach, N. Schnyder, C. Zimmermann, G. Imanidis, J. Drug Deliv. Sci. Technol., accepted.

[10] G. Imanidis, W. Zumbrunn, G. Di Pierro, Patent Cooperation Treaty (PCT) WO 2005/039685 A1, 6 May 2005. 